ISSN 1112-9867

http://www.jfas.info

\title{
IMPACT OF EARTHWORM ACTIVITY ON THE CHEMICAL FERTILITY OF IRRIGATED SOIL WITH URBAN EFFLUENTS
}

\author{
D. Addad ${ }^{1,2,3^{*}}$, M. Kribaa ${ }^{3}$, N. Ababsa ${ }^{2,3}$, L. Tamrabet ${ }^{3}$, M.Hafidi ${ }^{4}$, L. El Fels ${ }^{4}$, A. \\ Benmahammed $^{1}$ \\ ${ }^{1}$ Université Ferhat Abbas, Setif, ALGERIE \\ ${ }^{2}$ Université Abbes Laghrour, KHENCHELA, ALGERIE \\ ${ }^{3}$ Laboratoire RNAMS, Univ. LBM, Oum El Bouaghi, ALGERIE \\ ${ }^{4}$ Laboratoire Ecologie et Environnement. Université Cadi Ayyad Merrakech, MAROC
}

Received: 12 January 2017 / Accepted: 26 July 2017 / Published online: 01 September 2017

\begin{abstract}
The reuse of urban effluents to irrigate the soils of peri-urban grasslands in the vicinity of the town of Setif (northeastern Algeria) is an old and widespread practice. In this context, the present study was conducted to evaluate the effect of the irrigation with urban effluents on the biological and chemical behavior of soils. Effluents analysis showed significant organic and particulate pollution, the latter contributed to earthworm abundance and increased the richness of irrigated soils with nutrients. The analysis of turricules revealed the role of earthworms through the activity of bioturbation in the increase of the rate of organic matter as well as in the bioavailability of the nutrients of the irrigated soils. In space, permanent vegetation cover has played an important role as a biofilter. This was confirmed by the inter-site differences recorded through the measured variables particularly organic ones.
\end{abstract}

Keywords: Natural grasslands, urban effluents, earthworm activity, turricles, organic matter.

\footnotetext{
Author Correspondence, e-mail: dalath12@yahoo.fr

doi: http://dx.doi.org/10.4314/jfas.v9i3.5
} 


\section{INTRODUCTION}

L'épandage des eaux usées brutes en agriculture permet d'irriguer des cultures, d'épurer l'eau et de recycler des éléments nutritifs, le tout de manière économique. A la fin du $19^{\text {ème }}$ siècle, cette méthode avait souvent été mise en place autour des grandes villes pour éliminer les quantités croissantes d'eaux usées liées à l'urbanisation de la population [1]. Les eaux usées sont composées à $99 \%$ environ d'eau [2]. Dans une région semi-aride, une métropole d'un million d'habitants produirait suffisamment d'eaux usées pour irriguer approximativement 1500 à 3500 ha. En Algérie, leur volume annuel est estimé à 600 millions de $\mathrm{m}^{3}$, dont 550 millions de $\mathrm{m}^{3}$ correspond aux agglomérations de taille supérieure à 50000 habitants [3]. Les vers de terre, en tant qu'ingénieurs de sol, jouent un rôle important dans leur environnement grâce à différents mécanismes physico-chimiques et biologiques, permettant d'améliorer la fertilité et de préserver la structure du sol [4]. Les lombriciens modifient les propriétés physicochimique du sol créant ainsi une sphère d'influence particulière [5];[6]. Les turricules et les galeries constituent des microsites particuliers qui comparés au sol environnant présentent un enrichissement en $\mathrm{C}, \mathrm{N}$ et autres nutriments $(\mathrm{P}, \mathrm{K}, \mathrm{Mg}, \mathrm{NH} 4, \ldots)$ [7]. De plus les galeries creusées par les lombriciens constituent des voies préférentielles d'écoulement des eaux [8] entraînant ainsi divers particules [9]. Cette activité de bioturbation surtout des vers anéciques contribue au déplacement de la matière minérale du sol à partir des couches profondes vers la surface du sol [10]. Les turricules sont le siège de diverses réactions et processus physiques, chimiques et biologiques influençant ainsi le cycle biogéochimique de certains éléments majeurs tels que l'azote [11]; [7], le carbone [12]; [5], et le phosphore [13].

L'Objectif de cette étude est de comprendre l'effet des vers de terre, sur quelques paramètres chimiques d'un sol prairial irrigué par des rejets urbains bruts.

\section{EXPERIMENTAL}

\subsection{Milieu d'étude et échantillonnage}

Notre étude a été menée dans les prairies Pré-urbaines de la ville de Sétif, située au Nord-Est de l'Algérie. Ces prairies sont irriguées par les effluents urbains de cette ville. Les eaux usées 
de la ville de Sétif alimente oued Boussellam depuis les années soixante [14]. Cet oued qu'est le principal axe hydrographique de Sétif, alimente le barrage de Ain Zada.

Selon [15] les eaux usées brutes de la ville de Sétif représentent, non seulement, une source permanente et gratuite d'eau et de fertilisants, utilisées pour l'irrigation de l'espace prairial, mais aussi des répercussions graves sur la santé et sur l'environnement.

Le climat de la région est continental semi-aride, avec un été chaud et sec et un hiver froid et pluvieux. Les valeurs mensuelles moyennes des températures, sur une période de 36 ans (de 1981 jusqu'au 2015), passent de $24,80^{\circ} \mathrm{C}$ en été à $5,83^{\circ} \mathrm{C}$ en hiver. Les précipitations de la ville de Sétif sont irrégulières avec une moyenne annuelle de 402,03 $\mathrm{mm}$ pour la période (1981- 2011), ce qui implique forcement, une longue période de sècheresse qui s'étale, pour cette région de fin Mai jusqu'au début Octobrepace après.

Les sols de la région sont de texture argileuse à limono argileuse avec un taux élevé de calcaire.

Dans notre étude, nous avons choisi trois sites : Site 1 (S1) $\left(36^{\circ} 11^{\prime} 07.54^{\circ} \mathrm{N}, 5^{\circ} 23^{\prime} 03.36^{\prime \prime} \mathrm{E}\right.$;

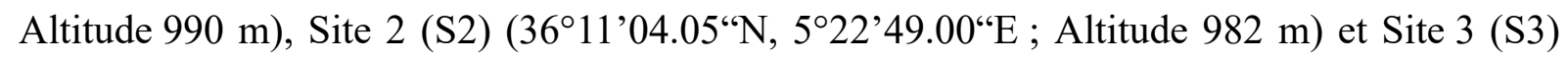
(36²'38.09“N $5^{\circ} 22^{\prime} 37.25^{\prime \prime E}$; Altitude $982 \mathrm{~m}$ ) de l'amont à l'aval à partir du point de rejet des eaux usées brutes de la ville de Sétif, en plus un site témoin $(\mathrm{T})\left(36^{\circ} 11^{\prime} 12.80^{\circ} \mathrm{N}\right.$ 5²3’10.86“E Altitude $1003 \mathrm{~m}$ ) témoin sans épandage des eaux usées. Ces trois sites sont choisis dans des prairies irrigués avec les eaux usées générées des agglomérations limitrophes de la ville de Sétif et déversées directement dans l'oued Boussellem (figure.1). 


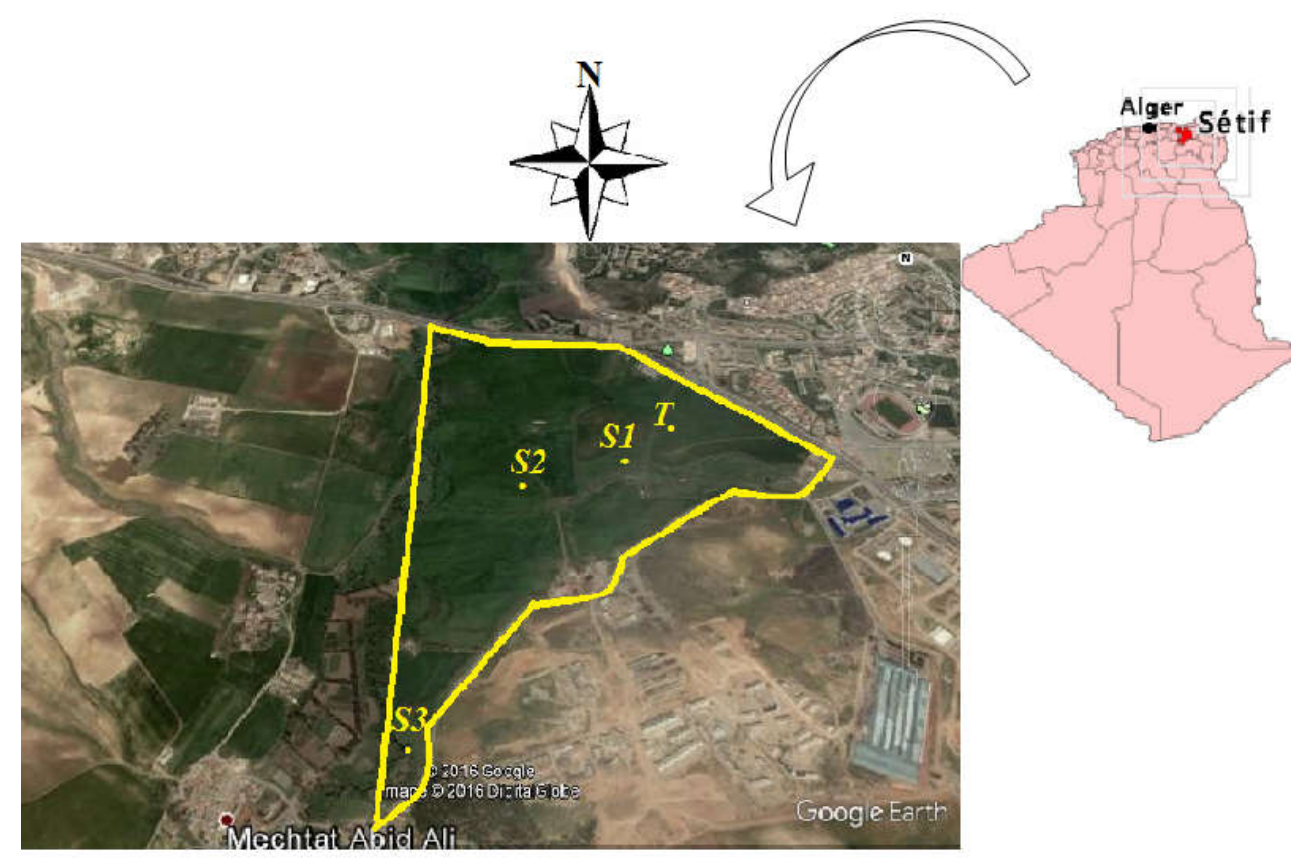

Fig.1. Image satellitaire des sites de prélèvement des échantillons de sol et turricules

(Source : Google Earth :16/04/2016)

Les échantillons des eaux sont prélevés, en Mars 2014, dans des bouteilles en polyéthylène de 1,5 litre au niveau des trois sites (3 répétitions par site) de l'amont vers l'aval du parcours des rejets urbains de la ville de Sétif.

Les prélèvements des sols (3 répétitions par site) sont effectués en Mars 2014 sur une profondeur de 7 à $20 \mathrm{~cm}$, alors que ceux des turricules (3 répétitions par site) sont récupérés à la surface de sol au niveau des quatre sites, les échantillons sont mis immédiatement dans des sachets en plastiques numérotés, puis séchés, broyés et tamisés.

\subsection{Méthodologie}

Pour les eaux usées, la caractérisation physico-chimique a été faite en mesurant la température, le $\mathrm{pH}$, la $\mathrm{CE}$ et $\mathrm{l}^{\prime} \mathrm{O}_{2}$ dissous qui ont été réalisés sur place à l'aide d'un multi paramètres. Les MES ont été mesurés par centrifugation [16], les chlorures, les nitrates, l'ammonium, les sulfates, les phosphates, le calcium et le magnésium sont analysées selon les méthodes décrites par Rodier et al. [17], la mesure de la $\mathrm{DBO}_{5}$ et de la $\mathrm{DCO}$ ont été effectuées à l'aide d'un DBO et DCO mètre respectivement et en fin le Na et $\mathrm{K}$ par spectrophotométrie à absorption atomique [17].

En ce qui concerne les sols et les turricules et pour bien apprécier le rôle des lombrics, nous 
avons mesuré quelques paramètres qui sont: le $\mathrm{pH}$ effectué avec un $\mathrm{pH}$ mètre sur une suspension de terre fine avec un rapport sol/eau de 1/2,5, le carbone organique total (COT) dont le carbone de la matière organique est oxydé par le bicarbonate de potassium en milieu sulfurique jusqu'à dégagement du $\mathrm{CO}_{2}$ l'excès de bichromate est titré par une solution de sel de Mohr en présence d'un indicateur: diphénilamine (méthode d'Anne décrite par [18]), la matière organique $(\mathrm{MO})$ est déduite à partir du carbone organique total en appliquant la formule $\mathrm{MO}=\mathrm{COT} \% \times 1,724$, la mesure de l'azote kjeldahl total $(\mathrm{N})$ est effectuée en deux étape : la première est la digestion de l'échantillon dans l'acide sulfurique concentré à des températures élevées pour convertir l'azote minéral sous forme ammoniacal et la deuxième consiste à déterminer l'ammonium dans l'extrait par titration de $\mathrm{NH}_{3}$ dégagé par la distillation à vapeur [18], l'extraction et le dosage des acides humiques $(\mathrm{AH})$ et fulviques $(\mathrm{AF})$ selon le schéma suivant. 


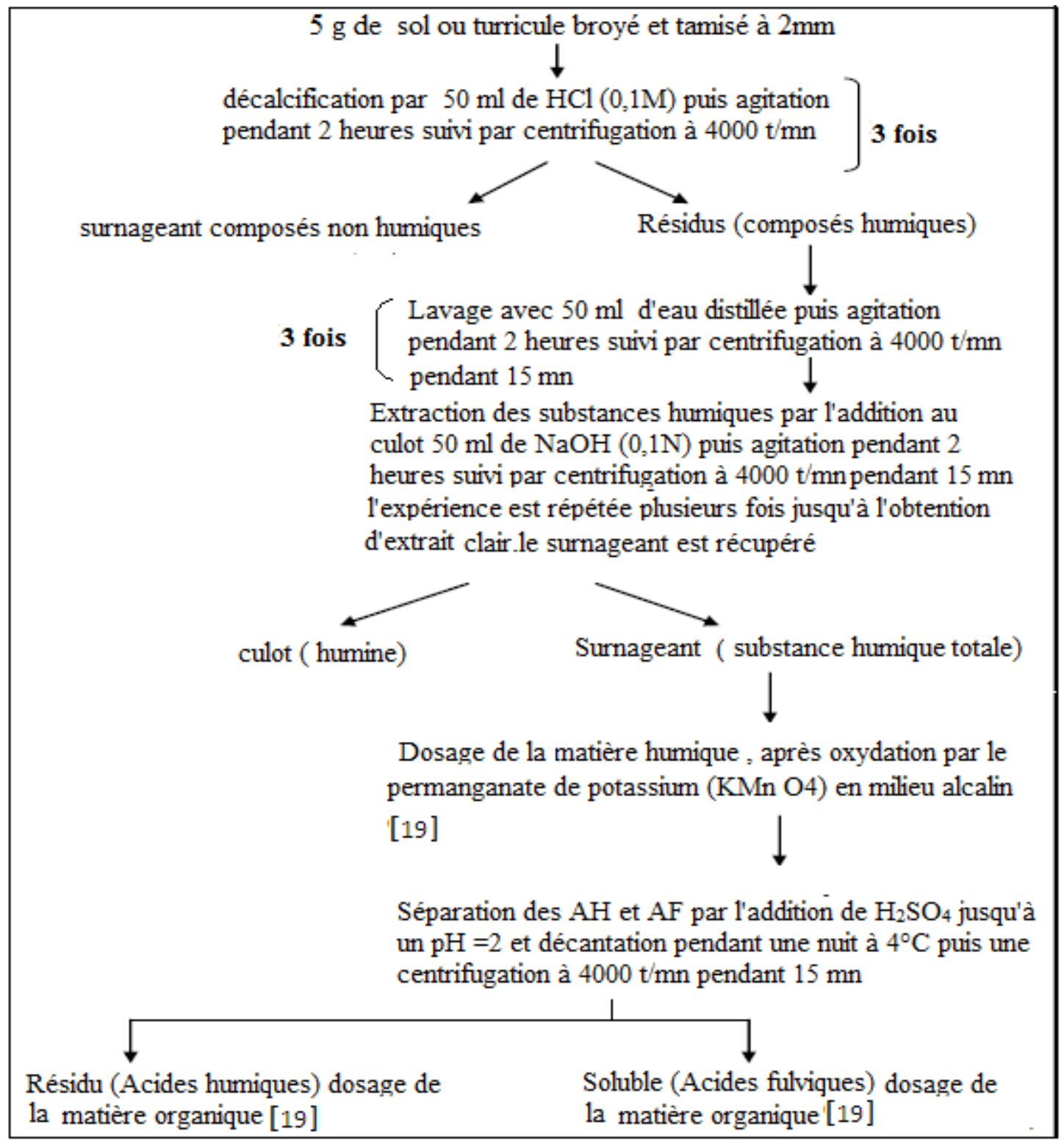

Fig.2. Schéma représentatif de l'extraction et dosage des acides humique et fulvique [19]

L'abondance lombricienne a été étudiée en utilisant la méthode de [20], suivi d'un tri manuel sur le volume d'action.

Pour valoriser nos résultats, nous avons mené une analyse statistique, il s'agit notamment de statistiques descriptives, l'analyse de variance (ANOVA), le test $\mathrm{t}$ de student ainsi une analyse en composantes principales en utilisant le logiciel statistique STATISTICA 08 et MNITAB 13.

\section{RESULTATS ET DISCUSSION}

\subsection{Caractérisation physico-chimique des eaux}


La qualité d'une eau est fonction des diverses substances qu'elle contienne, de leurs quantités et des effets qu'elles peuvent avoir sur l'écosystème et sur l'être humain.

Les résultats de l'analyse physico-chimique des eaux usées utilisés pour l'irrigation des prairies naturelles Sétifiennes sont mentionnés dans la figure (2)

L'examen de la composition physico-chimique des effluents urbains montre qu'elles ont, pour certains paramètres, des concentrations variables pouvant atteindre et dépasser des valeurs limites des normes internationales (FAO) de réutilisation des eaux usées en agriculture. Ceci est valable pour la CE, les chlorures, les MES, la DBO5 et la DCO. Alors que pour d'autres paramètres $\left(\mathrm{T}\left({ }^{\circ} \mathrm{C}\right), \mathrm{pH}, \mathrm{NO}_{3}^{-}, \mathrm{NH}_{4}^{+}, \mathrm{Ca}^{2+}, \mathrm{Mg}^{2+}, \mathrm{SO}_{4}^{2-}\right.$ et $\left.\mathrm{PO}_{4}^{3-}\right)$, les teneurs sont inferieures ou égales aux normes (FAO).

Les valeurs de la température des effluents urbains se situent dans une gamme favorable à l'activité microbienne $\left(<30^{\circ} \mathrm{C}\right)$. Ceci favorise le traitement biologique et l'auto-épuration des eaux usées [21] ainsi que le développement de la végétation [22]. Le pH des effluents urbains enregistre une légère diminution de l'amont vers l'aval, il varie de 8,4 à 8,3 et présente ainsi un caractère basique, ces valeurs ne dépassent pas la norme fixée par la FAO et adoptée par plusieurs pays du monde $(6,5$ à 8,4$)$. En ce qui concerne la conductivité électrique (CE) les valeurs enregistrées sont presque identiques pour les trois sites et dépassent largement la limite supérieure des directives de la FAO (3,0 dS/m), au-dessus de laquelle les eaux sont classées dans la catégorie « sévère restriction à l'usage ».

Contrairement aux autres paramètres, la matière en suspension (MES) et les chlorures $\left(\mathrm{Cl}^{-}\right)$ ont une tendance à augmenter de l'amont vers l'aval, les valeurs de ces derniers oscillent entre $596,4 \mathrm{mg} / 1$, valeur enregistrée en amont « S1 », et $710 \mathrm{mg} / 1$, valeur notée en aval « S3 », cette augmentation peut être expliquée par l'apport des rejets domestiques des habitants riverains et la nature des terrains traversés [23]; [24], ces valeurs dépassent largement la limite supérieure autorisée par la FAO (10 meq/1 ou $355 \mathrm{mg} / \mathrm{l})$ et peuvent atteindre les $200 \%$ de la norme dans le troisième site « $\mathrm{S} 3$ », pour les matières en suspension nous enregistrons une légère augmentation en passant de $0,539 \mathrm{~g} / 1$ en amont à $0,649 \mathrm{~g} / 1$ en aval. Les valeurs de la demande biologique en oxygène (DBO5) et la demande chimique en oxygène (DCO) montrent une légère diminution de l'amont vers l'aval, ces valeurs oscillent entre $239 \mathrm{mg} / \mathrm{l}$ et $232 \mathrm{mg} / \mathrm{l}$ pour 
la DBO5 et de $298,75 \mathrm{mg} / 1$ à $290 \mathrm{mg} / \mathrm{l}$ pour la DCO. En ce qui concerne les teneurs en nitrates, nous observons une diminution de l'amont vers l'aval, cette diminution passe de 1,93 $\mathrm{mg} / \mathrm{l}$, valeur enregistrée en amont, à $0,53 \mathrm{mg} / \mathrm{l}$, valeur notée en aval, ces valeurs là sont largement inferieures à la norme fixée par [25] (5 à $30 \mathrm{mg} / \mathrm{l})$. Les valeurs du sodium, calcium et magnésium des rejets urbain de la ville de Sétif restent dans les limites autorisées par la FAO (de 3 à 9 meq pour le sodium). A partir de ces trois paramètres $\left(\mathrm{Na}^{+}, \mathrm{Ca}^{2+}\right.$ et $\mathrm{Mg}^{2+}$, nous pouvons calculer le SAR (taux d'absorption du sodium) par la formule suivante: $\mathrm{SAR}=\left[\mathrm{Na}^{+}\right]$ / SQR $\left\{\left(\left[\mathrm{Na}^{+}\right]+\left[\mathrm{Mg}^{2+}\right]\right) / 2\right\}$, les valeurs obtenues en plus à la conductivité électrique nous ont permis de classer, selon le diagramme de Richards et Wilcox, les eaux des trois sites dans la catégorie $\mathrm{C}_{3} \mathrm{~S}_{1}$ dont l'eau est de qualité moyenne, à utiliser avec précaution, admissible pour l'irrigation des cultures tolérantes au sel, sur des sols bien drainés et où l'évolution de salinité doit cependant être contrôlée.

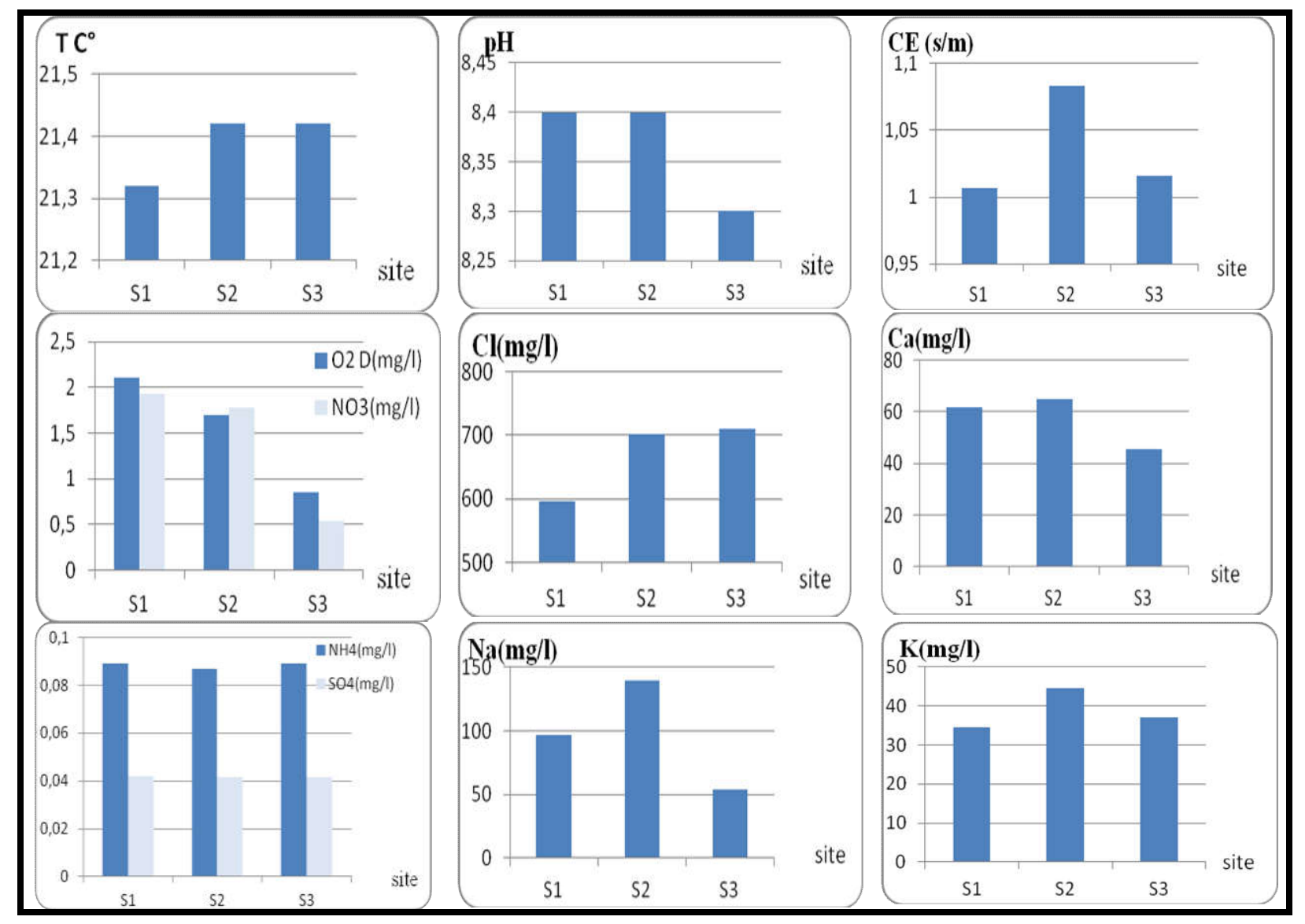




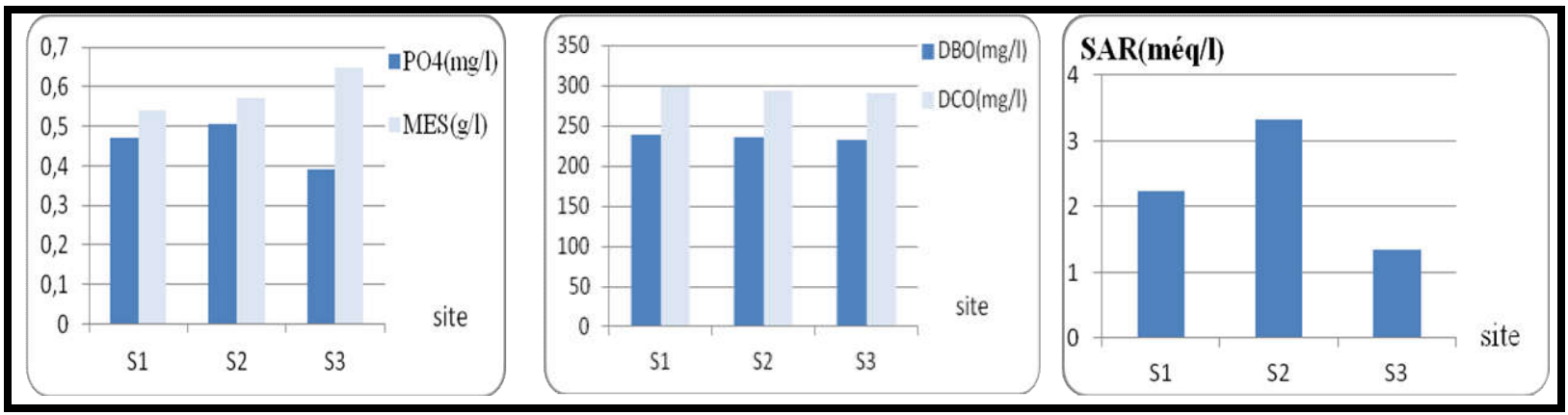

Fig.3. Résultats des analyses physico-chimiques des effluents urbains destinés à l'irrigation des trois sites ( $\mathrm{S} 1:$ amont, $\mathrm{S} 2:$ Mi axe et $\mathrm{S} 3:$ aval)

\subsection{L'abondance lombricienne}

Selon [26], les lombriciens modifient les propriétés physico-chimiques du sol, favorisant ainsi l'activité microbiologique.

En vue d'apprécier l'abondance lombricienne nous avons comparé les trois sites S1, S2 et S3 irrigués par les effluents de la ville de Sétif à un témoin qui reçoit seulement les eaux pluviales. Les résultats de cette comparaison sont représentés dans le tableau 1 suivant :

Tableau 1. Abondance lombricienne dans les sols des prairies irriguées avec les effluents urbains et le sol témoin

\begin{tabular}{rrrrr}
\hline Site & Adulte & Juvénile & Nombre total & $\begin{array}{r}\text { Augmentation en \% par rapport au } \\
\text { témoin }\end{array}$ \\
\hline S1 & 38 & 15 & 53 & 165 \\
S2 & 33 & 11 & 44 & 120 \\
S3 & 34 & 10 & 44 & 120 \\
T & 10 & 10 & 20 & - \\
\hline
\end{tabular}

Les lombriciens sont plus abondants dans le site S1 que les deux autres sites. Cette abondance arrive à $165 \%$ par rapport au témoin, les deux autres sites S2 et S3 présentent le même nombre de vers de terre et donnent une augmentation de $120 \%$ que le sol témoin. Cela révèle le rôle de l'irrigation par les eaux usées dans l'abondance lombricienne. La richesse des eaux usées en matières organiques et particulaire a favorisé l'abondance lombriciene dans les prairies [14]. Selon [27], les boues ne semblent pas avoir d'effet toxique direct ou indirect sur les lombriciens. De point de vu stade de développement des lombriciens, les sites exposés aux effluents urbains se caractérisent par des nombres de vers de terre adultes supérieurs que les juvéniles, alors que le site témoin représente le même nombre pour les deux stades. 
Pour les quatre sites nous avons dénombré identifié ; en utilisant la clé de Bouché [28] trois espèces de vers de terre appartenant à deux familles: Acanthodrilidae (Microscolex phosphoreus : espèce endogée) et Lumbrucidae (Allolobophora caliginosa : espèce endogée et Octodrilus lissaensis : espèce anécique).

\subsection{Effet des effluents urbains sur les sols des prairies}

Pour appréhender l'impact potentiel de l'irrigation avec les effluents urbains de la ville de Sétif sur les caractéristiques des sols des prairies pré urbaines, nous avons soumis les résultats des différents paramètres étudiés à une analyse de la variance (ANOVA), après avoir vérifié les conditions d'application de ce test et nous avons abouti au tableau suivant :

Tableau 2. Carré moyen de l'analyse de variance des paramètres mesurés

\begin{tabular}{llllllll}
\hline & $\mathrm{ddl}$ & $\mathrm{pH}$ & $\mathrm{C}(\%)$ & $\mathrm{MO}(\%)$ & $\mathrm{NKT}(\%)$ & $\mathrm{AF}(\mathrm{g} / \mathrm{Kg})$ & $\mathrm{AH}(\mathrm{g} / \mathrm{Kg})$ \\
\hline effet site & 3 & $0,00984^{\mathrm{ns}}$ & $19,73^{* *}$ & $58,76^{* *}$ & $0,09686^{* *}$ & $0,0615^{*}$ & $0,2036^{\mathrm{ns}}$ \\
Erreur & 8 & 0,00305 & 2,01 & 5,98 & 0,00533 & 0,0106 & 0,0520 \\
\hline ns, ${ }^{*}$ et ** : effet non significatif, significatif et hautement significatif respectivement \\
C : carbone total, MO : matière organique, NKT : azote kajeldahl totale, AF et AH : acides fulviques et humique \\
respectivement
\end{tabular}

A partir du tableau (1) nous pouvons apprécier que l'irrigation avec les eaux usées montre un effet significatif à hautement significatif pour l'ensemble des paramètres étudiés sauf pour le $\mathrm{pH}$ et les acides Humiques qui ont un effet non significatif.

Les valeurs moyennes du $\mathrm{pH}$ des sols étudiés varient entre 7,83 pour le site 2 et 7.97 pour le site témoin (figure 3). Nous enregistrons ainsi une faible diminution de $\mathrm{pH}$ des sites irrigués avec les effluents urbains par rapport au site témoin (non irrigué), rappelant que cette diminution n'est pas significative de point de vue statistique. Plusieurs chercheurs mentionnent que le $\mathrm{pH}$ du sol irrigué avec les eaux usées décroît suite à l'oxydation des composés organiques et la nitrification de l'ammonium [29] ; [30] ; [31]. Des résultats similaires ont été aussi rapportés par [32]. Selon [33], un pH de sol légèrement basique empêche modérément la minéralisation et la dissolution des nutriments pour les rendre disponibles aux racines des plantes. 


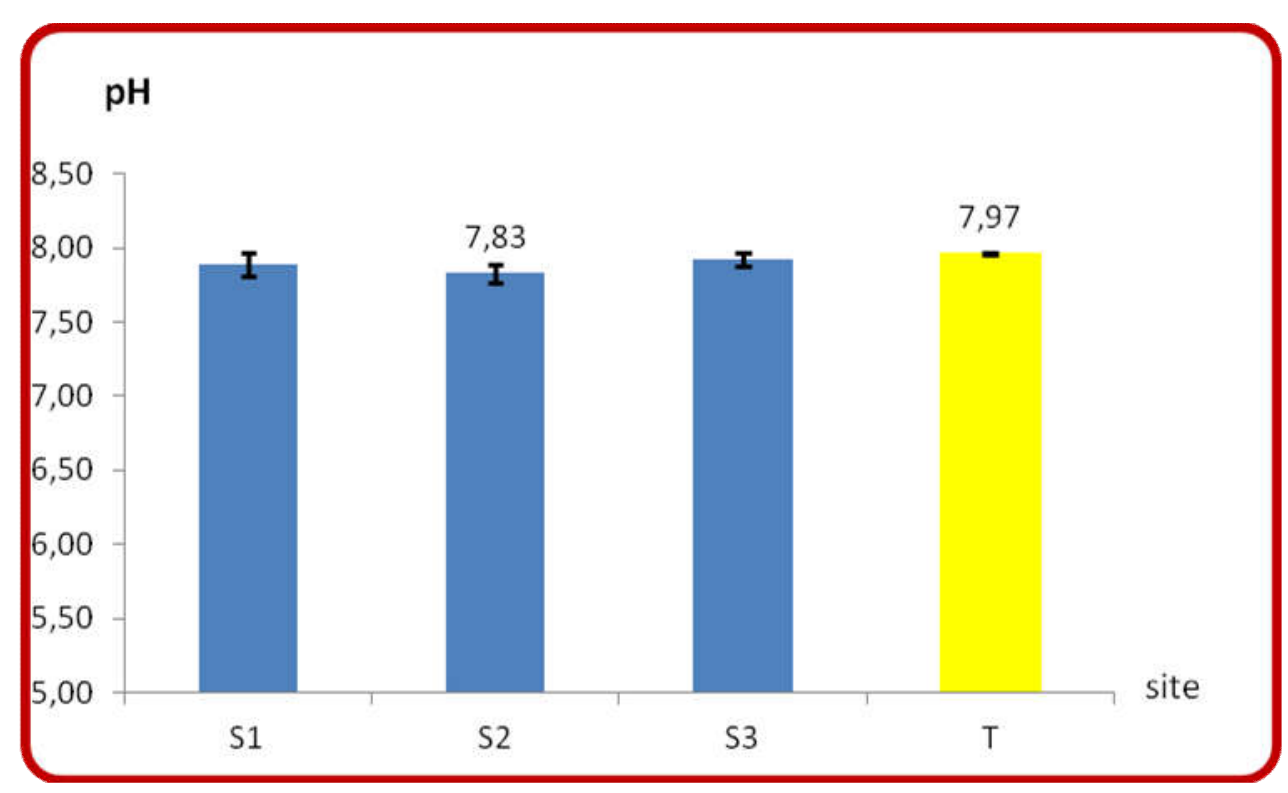

Fig.4. Variation du pH des sites irrigués avec des effluents urbains par rapport au site témoin

En ce qui concerne la matière organique, l'analyse de la variance a révélé un effet irrigation par les eaux usées hautement significatif, ce qui a été traduit pratiquement par la comparaison des moyennes, où nous avons enregistré des augmentations de la teneur du matière organique dans les trois sites, ces augmentations par rapport au site témoin, passent de plus de six fois pour les site S1 et S2 à presque huit fois plus pour le site S3 aval (Figure 4). Cet effet est le résultat de la teneur élevée des eaux usées utilisées comme substrat d'irrigation en composés organiques (valeurs élevées de la DBO et la DCO des effluents urbains) ce qu'a été approuvé par plusieurs auteurs comme [34], [29] et par [35].

Selon [36] et [37] les sols soumis à l'irrigation avec les eaux usées traitées ou brutes se distinguent par une amélioration du taux de MO, ce qui affecte l'agrégation du sol, les réserves en minéraux et leur disponibilité ainsi que l'activité biologique. 


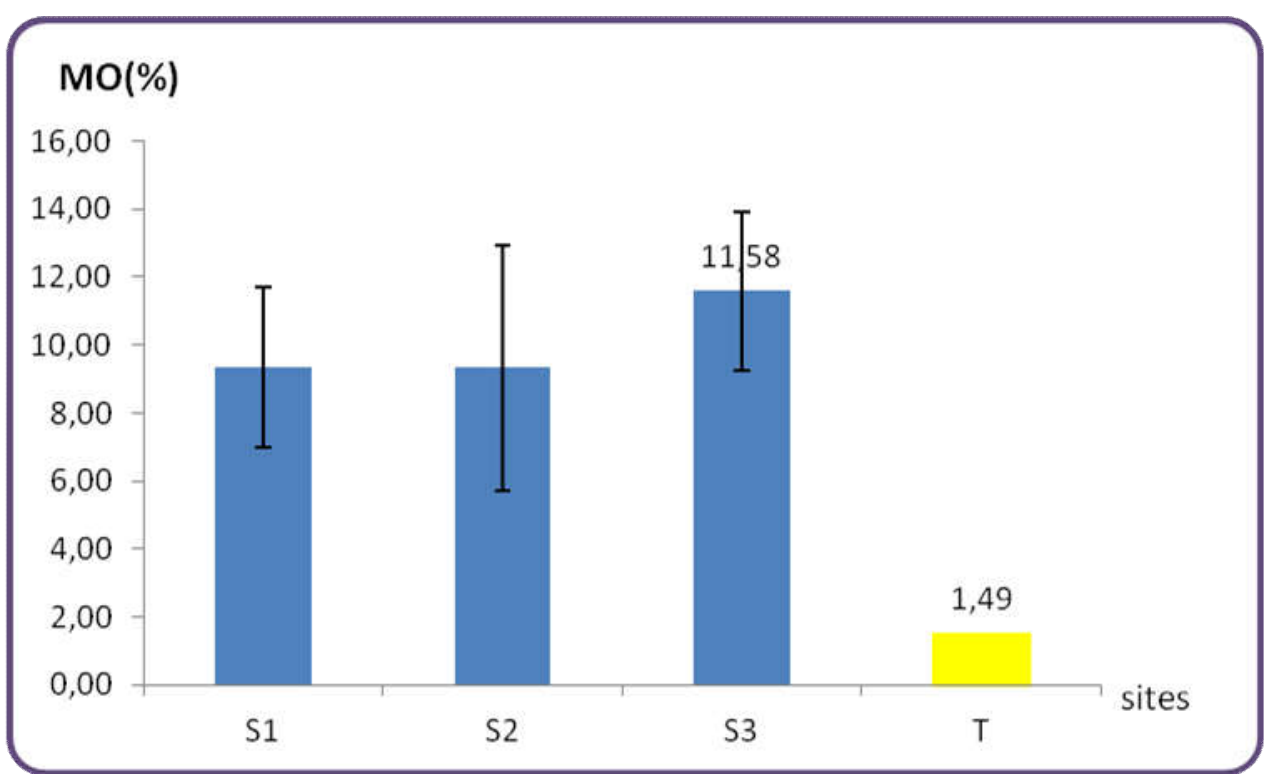

Fig.5. L'effet moyen de l'irrigation avec les effluents urbains sur la matière organique

Les valeurs moyennes de l'azote total kjeldahl (NKT) des sites étudiés oscillent entre 0,20\%, valeur observée pour le site témoin, et $0,63 \%$, valeur moyenne du site $\mathrm{S} 3$, enregistrant ainsi une augmentation de trois fois plus que la valeur du site témoin, ce qui révèle un effet significatif de l'irrigation avec les effluents urbains sur la teneur en azote des sols irrigués (fig. 5). Nos résultats sont similaires à ceux rapportés par [38], [39], [40] et [35].

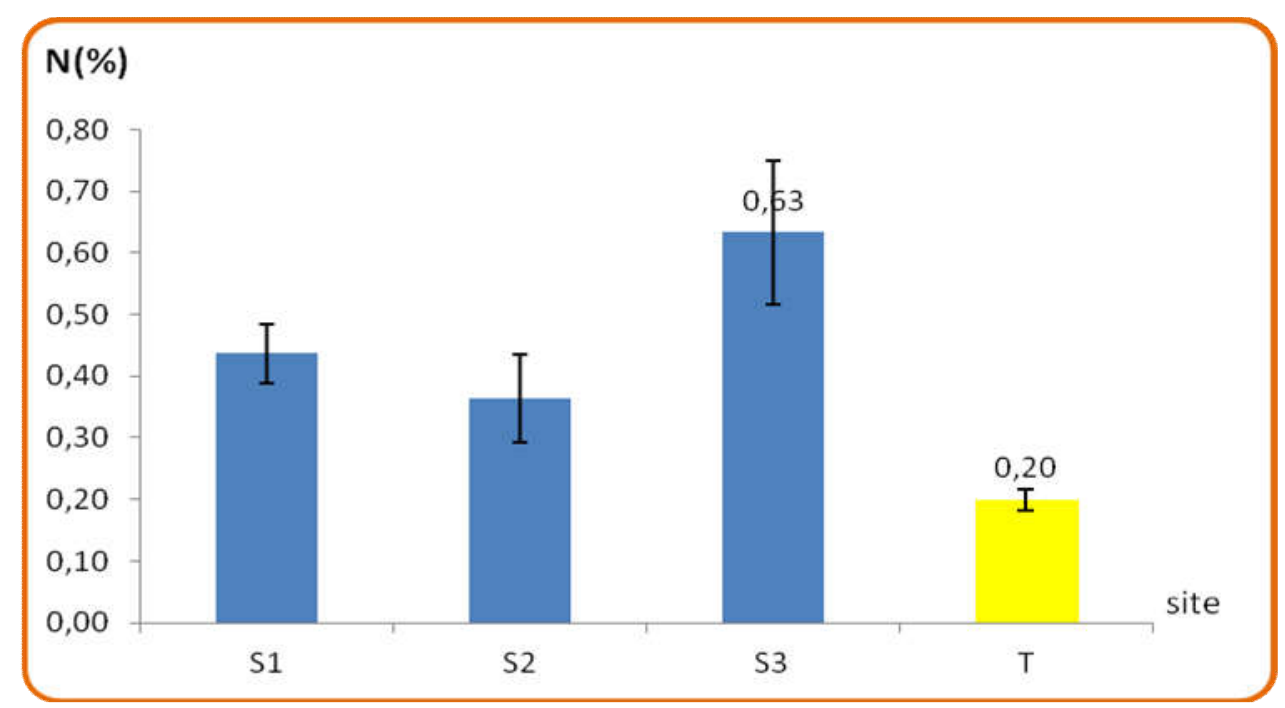

Fig.6. La variabilité de l'azote Total dans les sites irrigués avec des effluents urbains par rapport au témoin

Les teneurs de la matière organique et de l'azote sont fortement corrélées $(r=0,806)$, ce qui suppose qu'elles ont la même source (eau usée). 
La nette différence entre la concentration en carbone total du sol témoin et les sols des prairies irrigués par les effluents urbains est aussi illustrée par les teneurs en acides fulviques et humiques largement différentes (Tableau 2). Nous trouvons, en effet, dans le site S3 (point d'aval) $0,38 \%$ d'acide fulvique et $0,54 \%$ d'acide humique soit plus de 3 et 7 fois des valeurs enregistrées au niveau du sol témoin, alors que le site S1 (point d'amont) enregistre la valeur la plus élevée des acides fulviques, qui est de $0,42 \%$ et $0,25 \%$ des acides humiques soit 3,5 fois plus que celles mesurées au niveau du sol témoin, nous remarquons également une teneur élevée des acides humiques pour le site S2 (Mi) (Tableau 2).

Tableau 3. Teneurs (\%) des sols en acides fulviques et humiques et l'indice d'humification (IH)

\begin{tabular}{lrrrr}
\hline Site & $\mathrm{C}(\%)$ & $\mathrm{AF}(\%)$ & $\mathrm{AH}(\%)$ & $\mathrm{IH}=\mathrm{AH} / \mathrm{AF}$ \\
\hline S1(amont) & 5,42 & 0,42 & 0,25 & 0,61 \\
S2 (mi) & 5,42 & 0,18 & 0,63 & 3,45 \\
S3 (aval) & 6,72 & 0,38 & 0,54 & 1,44 \\
T & 0,87 & 0,12 & 0,07 & 0,55 \\
\hline
\end{tabular}

La proportion des acides fulviques est supérieure à celle des acides humiques dans les deux sites $\mathrm{S} 1$ et $\mathrm{T}$ soit dans la moitié des échantillons situés tous dans l'amont du site d'étude, les deux autres sites représentent des teneurs plus élevées des acides humiques. Cette répartition permet de constater qu'il y a plus d'acides humiques dans les points à forte teneur en matière organique cela concorde avec les résultats de [41].

L'indice de maturité de la matière organique humifiée exprimé par le rapport: Acides humiques/Acides fulviques, indique une faible maturité si sa valeur est inferieur à 1 et une forte maturité s'il est supérieur à 1,3 [42]. Les sites S2 et S3 se caractérisent par des matières organiques à haut niveau de maturité, alors que les autres sites sont à faible niveau de maturité.

\subsection{Impact de l'activité lombricienne sur la qualité des sols irrigués avec les effluents urbains}

Les vers de terre sont capables d'ingérer de grandes quantités de terre. Ainsi, un individu peut consommer jusqu'à 35 fois son propre poids de terre [43], ce sol ingérés est expulsé par la suite sous forme de turricule dans ou à la surface de sol. Pour bien éclairer l'effet des lombrics 
sur la composition chimique de sol nous avons choisi les turricules pour les comparer avec le sol environnant. Les résultats du test $t$ pour les paramètres étudiés sont donnés dans le tableau (4).

Tableau 4. L'effet moyen des lombrics sur quelques paramètres de sol

\begin{tabular}{|c|c|c|c|c|c|c|c|c|c|c|c|c|}
\hline \multirow[t]{2}{*}{ Paramètres } & \multicolumn{2}{|c|}{$\mathrm{pH}$} & \multicolumn{2}{|c|}{$\mathrm{C}(\%)$} & \multicolumn{2}{|c|}{$\mathrm{MO}(\%)$} & \multicolumn{2}{|c|}{ NKT(\%) } & \multicolumn{2}{|c|}{$\mathrm{AF}(\%)$} & \multicolumn{2}{|c|}{$\mathrm{AH}(\%)$} \\
\hline & sol & tur & Sol & tur & sol & tur & Sol & tur & sol & tur & sol & tur \\
\hline Moy & 7,88 & 7,07 & 5,85 & 10,21 & 10,09 & 17,61 & 0,48 & 0,76 & 0,33 & 1,30 & 0,48 & 1,67 \\
\hline $\begin{array}{ll}\text { Moy } & \text { Tur-Moy } \\
\text { Sol } & \end{array}$ & \multicolumn{2}{|c|}{$-0,81$} & \multicolumn{2}{|c|}{4,36} & \multicolumn{2}{|c|}{7,52} & \multicolumn{2}{|c|}{0,29} & \multicolumn{2}{|c|}{0,97} & \multicolumn{2}{|c|}{1,19} \\
\hline Valeur de $t$ & \multicolumn{2}{|c|}{23,57} & \multicolumn{2}{|c|}{$-3,90$} & \multicolumn{2}{|c|}{$-3,90$} & \multicolumn{2}{|c|}{$-4,62$} & \multicolumn{2}{|c|}{$-6,70$} & \multicolumn{2}{|c|}{$-9,08$} \\
\hline Probabilité & \multicolumn{2}{|c|}{0,000} & \multicolumn{2}{|c|}{0,002} & \multicolumn{2}{|c|}{0,002} & \multicolumn{2}{|c|}{0,001} & \multicolumn{2}{|c|}{0,000} & \multicolumn{2}{|c|}{0,000} \\
\hline Aug/T Sol(\%) & \multicolumn{2}{|c|}{$-10,26$} & \multicolumn{2}{|c|}{74,57} & \multicolumn{2}{|c|}{74,56} & \multicolumn{2}{|c|}{59,74} & \multicolumn{2}{|c|}{299,45} & \multicolumn{2}{|c|}{250,06} \\
\hline
\end{tabular}

Moy : moyenne, Tur : turricule,

Aug/T Sol(\%) : augmentation par rapport à la teneur du sol (\%)=[(Moy Tur-Moy Sol)/ Moy sol]x100

Les différences entre les moyennes des turricules et celles des sols sont hautement à très hautement significatives pour l'ensemble des variables mesurées. Le pH des turricules est inferieur à celui du sol, cela révèle l'effet neutralisant exercé par les lombrics sur le sol tout en passant d'un milieu faiblement alcalin à un milieu neutre. Cet effet est observé dans les trois sites (figure 6). [44] rappelle que les vers de terre stabilisent le $\mathrm{pH}$ du sol. Les teneurs moyennes en Carbone organique total et en Azote Kjeldahl Total sont significativement plus élevées dans les turricules que dans le sol environnant (tableau 4). De nombreux auteurs [45]; [12] ; [11] ; [46] ; [47] ; [26] ont observés une augmentation des concentrations du carbone total dans les turricules. Les teneurs de $\mathrm{C}$ et de $\mathrm{N}$ dans les déjections peuvent être, selon [12] de 1,5 à 2,0 fois et selon [26] de 2 à 3 fois supérieures à celles du sol environnant. Dans notre étude les teneurs de carbone et d'azote des turricules sont de 1,75 et 1,60 fois supérieures à celles mesurées pour le sol environnant. Les augmentations de la matière organique des turricules par rapport au sol environnant passent de 0,08\% dans le site S3 à 14,57 \% dans le site S1 (figure 7). Ces augmentations oscillent, pour l'azote entre 0,027\%, valeur enregistrée dans le site S3 et 0,491\%, valeur marquée dans le site S2. Cet enrichissement en C et $\mathrm{N}$ des microsites, illustré par de nombreux auteurs, serait du à l'ingestion préférentielle des particules organiques par les vers [12] ; [48]. En ce qui concerne les acides fulvique et 
humique, nous constatons que les turricules révèlent des teneurs plus importantes que celles des sols. Les différences en entre les moyennes des turricules et celles du sol sont de l'ordre de $0,97 \%$ et $1,19 \%$ pour les acides fulviques et humiques respectivement, ce qui donne une augmentation moyenne presque de $300 \%$, pour les acides fulviques et $250 \%$, pour les acides humiques. De point de vue site, nous remarquons que dans le site S1, les turricules contiennent de l'acide fulvique 2,5 fois plus que le sol environnant, cette valeur arrive à 8,5 fois dans le site S2, pour les acides humiques, les turricules sont plus chargées que le sol environnant de 2,5 fois pour le site $\mathrm{S} 2$ à 8,5 fois pour le site $\mathrm{S} 1$ (figure 9). L'activité biologique élevée favorise probablement la formation des substances humiques condensées solubles dans l'extrait alcalin avec une plus grande stabilité [49].

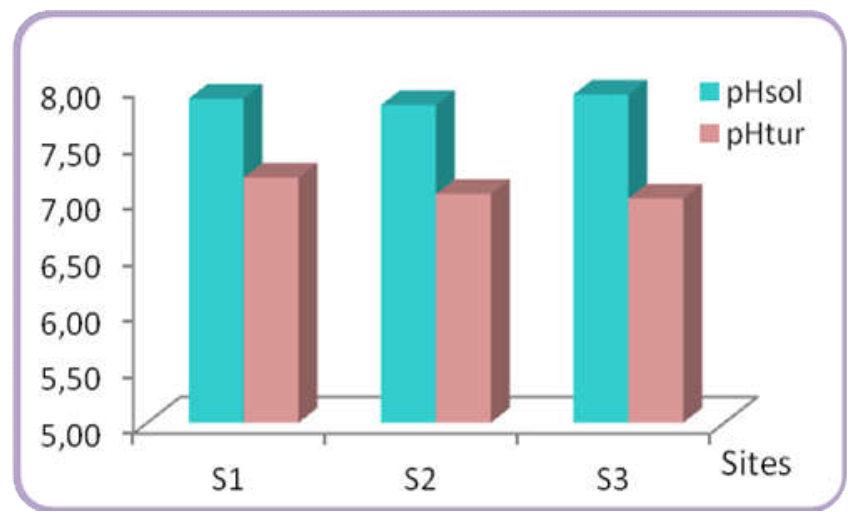

Fig.7. Comparaison entre le $\mathrm{pH}$ de sol et celui de turricule

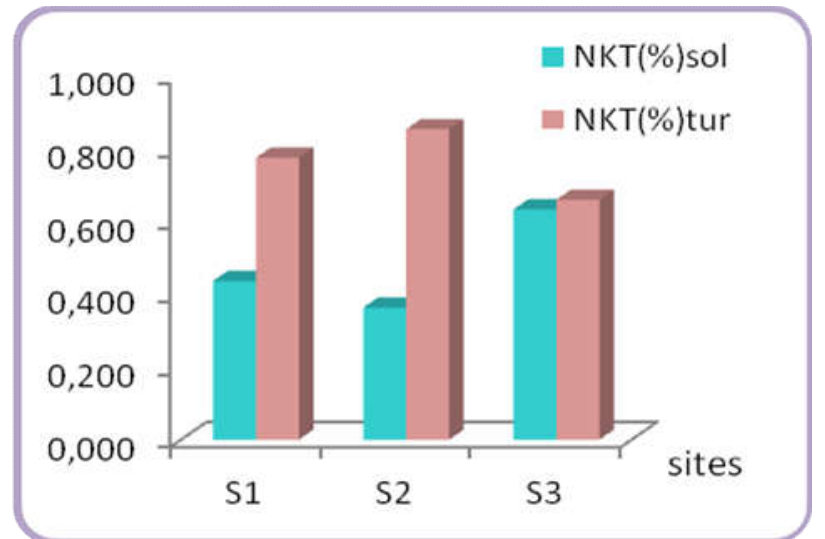

Fig.9. Comparaison entre l'azote kjeldahl total de sol et celui de turricule

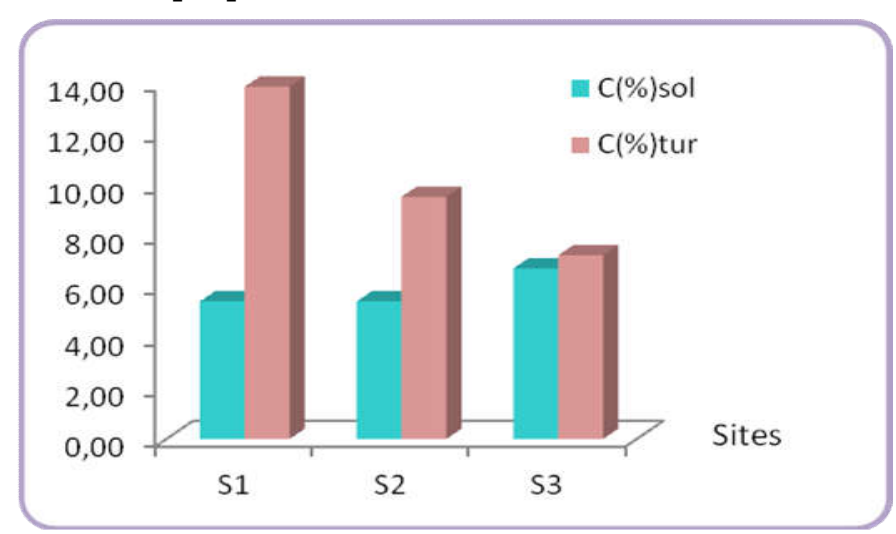

Fig.8. effet moyen des lombrics sur le carbone et la matière organique de sol

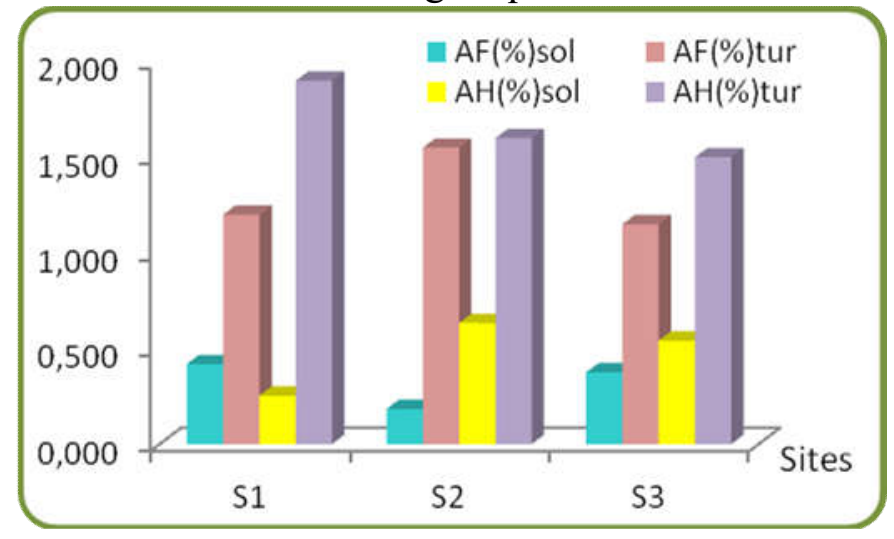

Fig.10. effet moyen des lombrics sur les acides humiques et fulviques de sol

Pour l'indice d'humification la différence des moyennes entre le sol et les turricules n'est pas significative $(\mathrm{P}=0,708)$. 


\subsection{Liaisons inter-caractères}

L'étude des corrélations entre les différentes paires de caractères est menée pour identifier les caractères qui évoluent dans le même sens et ceux qui s'opposant. Parmi les liaisons les plus intéressantes, nous pouvons citer les corrélations négatives du $\mathrm{pH}$ avec l'azote $(\mathrm{r}=-0,800)$, l'acide fulvique $(\mathrm{r}=-0,955)$ et l'acide humique $(\mathrm{r}=-0,940)$, les corrélations positives de la matière organique avec les mêmes variables $\left(\mathrm{r}_{\mathrm{MOxNKT}}=0,875 ; \mathrm{r}_{\mathrm{MOxAF}}=0,771\right.$ et $\left.\mathrm{r}_{\mathrm{MOxAH}}=0,885\right)$, la corrélation positive entre l'azote et l'acide humique $(\mathrm{r}=0,867)$ et celle entre les acides humique et fulvique $(r=0,917)$.

La recherche de liaisons est étudiée grâce à l'analyse en composante principale ACP, les deux premiers axes détiennent respectivement $71,35 \%$ et $14,35 \%$ d'information. L'axe 1 de l'ACP intègre l'information apportée par les paramètres : matière organique, azote kjeldal total, les acides humiques et fulviques et l'abondance lombricienne d'une part et d'autre part, le $\mathrm{pH}$. Ce dernier paramètre qui est positivement corrélé à l'axe 1 s'oppose aux autres paramètres tout le long de cet axe et qui lui sont négativement corrélés. Alors que l'indice d'humification est négativement lié à l'axe 2 de l'ACP (figure.10).

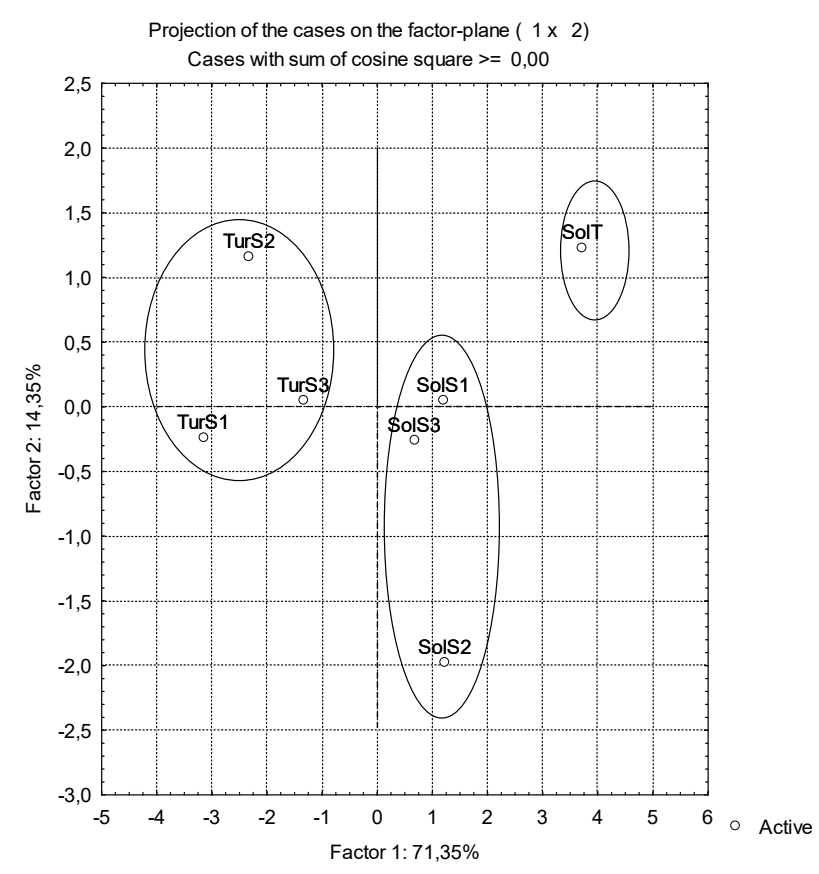

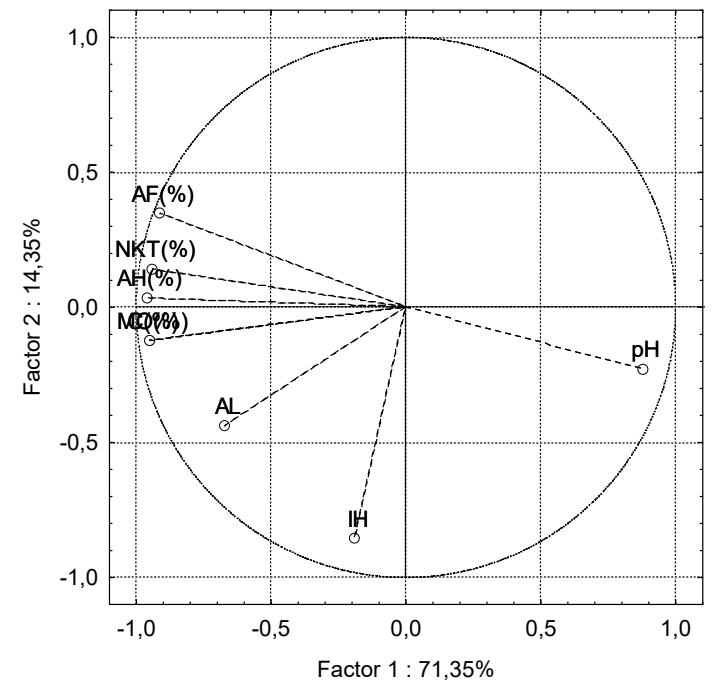

Active

Fig.11. projection des paramètres mesurés sur le plan $(1,2)$ de l'analyse en composantes principales
Fig.12. projection des individus mesurés sur le plan $(1,2)$ de l'analyse en composantes principales 
La distribution des individus nous a permis d'élaborer 3 groupes, le groupe 1 formé par les turricules des trois sites est négativement corrélé à l'axe 1 et le groupe 2 formé par le sol témoin est positivement corrélé au même axe, ces deux groupes s'opposent tout le long de cet axe, et enfin le groupe 3 formé par les sols irrigués par les effluents urbains de la ville de Sétif qu'est négativement corrélé à l'axe 2 de l'ACP (figure 11).

L'ACP a montré l'effet significatif des lombrics sur la concentration et la redistribution des éléments nutritifs, où nous avons remarqué que les turricules contiennent plus de matière organique, d'acides humique et fulviques et d'azote que les sol irrigués par les eaux usées et beaucoup plus que le sol témoin, ce dernier se caractérise par un $\mathrm{pH}$ élevé. L'effet de l'irrigation par les effluents urbains, sur les paramètres mesurés, est bien clair au niveau des trois sites comparativement au témoin.

\section{CONCLUSION}

Notre étude a été menée dans le but d'apprécier l'effet des lombrics sur la qualité physicochimique et biologique d'un sol irrigué par les effluents urbains. Les résultats d'analyse des eaux usées de la ville Sétif montrent une pollution organique et particulaire assez élevée. Cette charge organique a favorisé l'abondance lombricienne au niveau des sols irrigués par les effluents, où elle peut atteindre plus de 2,5 fois que celle du sol témoin. La plupart des paramètres du sol sont significativement affectées par l'irrigation aux effluents urbains et le sol s'est amélioré en fertilité. L'effet de l'activité lombricienne est étudié en comparant les turricules aux sols environnants. Les teneurs des turricules en éléments chimiques sont supérieures, significativement, à celles obtenues dans le sol.

\section{RÉFÉRENCES}

[1] Filip G.M., Fitzgerald S.A. \& Ganio LM. Precommercial thinning in a ponderosa pine stand affected by Armillaria root disease in central Oregon: 30 years of growth and mortality. West.]. Appl. For, 1999, 14(3):144-148.

[2] OMS. Directives OMS pour l'utilisation sans risque des eaux usées, des excréta et des eaux ménagères, volume II : Utilisation des eaux usées en agriculture Éditions de l'OMS, Organisation mondiale de la Santé, Genève 27 (Suisse)., 2012, p 225. 
[3] MRE. Le secteur de l'eau en Algerie. Ministere des Ressources en Eau, Algérie, 2003.

[4] Lavelle, P., Bignell, D., Lepage, M., Wolters, V., Roger, P., Ineson, P., Heal, O.W., Ghillion, S. 1997. Soil function in a changing world: The role of invertebrate ecosystem engineers. Eur. J. Soil Biol. 33, 159-193.

[5] Shuster W.D., Subler S. \& McCoy E.L. 2001. Deep-burrowing earthworm additions changed the distribution of soil organic carbon in a chisel-tilled soil. Soil Biol. and Biochem. 33 : 983-996. doi: 10.1016/S0038-0717(01)00002-5

[6] Tiunov A.V. \& Scheu S. 1999. Microbial biomass, biovolume and respiration in Lumbricus terrestris casts materail of different age. Soil Biol. Biochem. 32(2) :265:275. doi: 10.1016/S0038-0717(99)00165-0

[7] Parkin T.B. \& Berry E.C. 1999. Microbial nitrogen transformations in earthworms burrows. Soil Biol. Biochem. 31(13) : 1765-1771. doi: 10.1016/S0038-0717(99)00085-1

[8] Capowiez, Y., Cadoux, S., Bouchant, P., Ruy, S., Roger-Estrade, J., Richard, G. and Boizard, H., 2009. The effect of tillage type and cropping system on earthworm communities, macroporosity and water infiltration. Soil Tillage Res., 105(2): 209-216.

doi: 10.1016/Jj.still.2009.09.002.

[9] Shipitalo M.J., Edwards W.M., Redmond C.E. Comparison of water movement and quality in earthworm burrows and pan lysimeters. J. Environ. Qual., 1994, 23:1345-1351.

[10] Asshoff R., Scheu S. and Eisenhauer N. 2009. Different earthworm ecological groups interactively impact seedling establishment. European Journal of Soil Biology, 46:330-334.

[11] Binet F. Dynamique des peuplements et fonctions des lombriciens en sols cultivés tempérés. Thèse doctorat Université, Rennes I., 1993, 299 p.

[12] Lee K.E. Earthworms. Their Ecology and relationship with soils and land use. Academic Press, Sydney, 1985,411 p.

[13] Le Bayon C. Influence des activités des lombriciennes sur la dynamique (disponibilité, transfert) du phosphore en sols tempérés. Thèse de doctorat, Université de Rennes 1.,1999, $133 \mathrm{p}$. 
[14] Ababsa, N. Identification et quantification in situ des interactions entre la diversité faunique et la macro prairies naturelles irriguées à l'eau usée.fonctionnement hydrique du sol. Thèse de doctorat. Université de Sétif. 2016. 129 p.

[15] Ababsa N., Kribaa M., Addad D., Tamrabet L and Baha M. 2016. Does earthworms density really modify soil's hydrodynamic properties in irrigated systems with recycled water. Journal of Fundamental and Applied Sciences, 8(2), 627-638. doi:10.4314/jfas.v8i2.29

[16] Rejsek, F. Analyse des eaux, Aspects réglementaires et techniques. Scérén TEC \& DOC. CRDP Aquitaine., 2002. 358 p.

[17] Rodier, J., Bernard, L., Nicole, M., et coll. L'analyse de l'eau. Eaux naturelles. Résiduaires. Eau de mer. 9ème édition. Dunod. Paris, 2009, 1526p.

[18] Aubert G. Méthodes d'analyses des sols. CRDP Marseille, 1978, 191 p.

[19] Chaminade R. Les formes de phosphore dans le sol. Nature et rôle des complexes phospho-humiques. Ann. Agron, XIV, ,1944, 1-52.

[20] Raw F. Estimating earthworm population by using formaline. Nature, 1959,184, 1661-1662.

[21] Mara D.D. Sewage treatment in hot climate. Editions John Wiley \& Sons, New York. 1980.

[22] Marschner H. Mineral nutrition of higher plants. 2nd edition. Academic press, 1995.

[23] Rodier J. Analyse de l'eau : Eaux naturelles, Eaux résiduaires, Eau de mer. Edition Dunod, Paris., 1996, 1384 p.

[24] Bermond R. et Vuichard R. Paramètres de la qualité des eaux. Ministère de la protection de la nature et de l'environnement. SPEPE, Paris., 1973, 179 p.

[25] FAO. L'irrigation avec des eaux usées traitées : Manuel d'utilisation. 2003, p 73

[26] Lavelle P. 1997. Faunal activities and soil processes: adaptative strategies that determine ecosystem function. Advances in ecological soil research. 27: 93-132.

Doi : 10.1016/S0065-2504(08)60007-0

[27] Ablain F. Rôle des activités lombriciennes sur la redistribution des éléments traces métalliques issus de boue de station d'épuration dans un sol agricole. Thèse Doc. Ecole 
doctorale Vie-Agro-Santé. U.F.R Sciences de la vie et de l'environnement. Univ. Renne France., 2002, p 148.

[28] Bouché, M.B. Lombriciens de France, écologie et systématiques.INRA, Paris. 1972. 671

p.

[29] Mohammad, M.J. \& Mazahreh, N. 2003. Changes in soil fertility parameters in response to irrigation of forage crops with secondary treated wastewater. Soil Sci. Plant Anal. 34(9\&10):1281-1294. doi:10.1081/CSS-120020444.

[30] Vazquez Montiel, O., Horan, N.J. and Mara, D.D. Management of domestic wastewater for reuse in irrigation. Water Sci. Technol., 1996, 33(10-11) 355-365.

[31] Hayes, A.R., Mancino, C.F. and Pepper, I.L. 1990. Irrigation of turfgrass with secondary sewage effluent. I. Soil and leachate water quality. Agron. J. 82:939-943. doi:10.2134/agronj1990.00021962008200050021x

[32] Alhands, M.N., Allick, S.A., Overman, A.R., Lesema n, W.G. and Vidak, W. 1995. Municipal water use at Tallahassee, Florida. Trans. ASAE 38(2):411-418. doi:10.13031/2013.27847.

[33] Benlkhoubi N, Saber S, Lebkiri A, Rifi El and Fahime El. 2016. Evaluation of physico-chemical parameters of agricultural soils irrigated by the waters of the hydrolic basin of Sebou river and their influences on the transfer of trace elements into sugar crops (the case of sugar cane). J.Fundam. Appl. Sci. 8(2), 438-451. doi.org/10.4314/jfas

[34] Mancino, C.F. and Pepper, I.L. Irrigation of turfgrass with secondary sewage effluent: Soil quality. Agron. J., 1992, 84(4):650-654.

doi:10.2134/agronj1990.00021962008400040023x

[35] Tamrabet L. Contribution à l'étude de la valorisation des eaux usées en maraichage. Thèse doc. Univ. Batna., 2011, 146 p.

[36] Hillel, D. Environmental soil physics: fundamentals, applications and environmental considerations. Academic Press, 1998, 801 p.

[37] Sparks, L.D. Environmental soil chemistry. Academic Press, 2nd Edition, 2003, 367p.

[38] Reddy, K. R., And, W. H. \& Patrick, Jr. Losses of applied ${ }^{15} \mathrm{NH}_{4}-\mathrm{N}$, urea ${ }^{15} \mathrm{~N}$, and organic $\mathrm{N}$ in flooded soils. Soil Sci. , 1980, 130: 326-330. 
[39] Liu, HY., Badarinarayana, V., Audino, D.C., Rappsilber, J., Mann, M. \& Denis C.L. The NOT proteins are part of the CCR4 transcriptional complex and affect gene expression both positively and negatively. EMBO J, 1989, 17(4):1096-106.

[40] Midrar, U.H., Khattak, R.A., Puno, H.Kh. \& Saleem-Saif, M. 2004. NPK status in effluent irrigated soils of some selected sites of NWFP. Int. J. Agric. Biol. 6(2): 264-267.

[41] Ben Hassine, H., Karbout, N., Kridane, K., Sanaa, M. \& Jedidi, N. 2012. Caractérisation des fractions colloïdales minérales et organiques des horizons superficiels des sols d'une toposéquence en zone semi-aride de la Tunisie. Etude et gestion des sols, 19, 2 : 105-118.

[42] Francou S. 2003. Stabilisation de la matière organique au cours du compostage des déchets urbains : influence de la nature des déchets et du procédé de compostage - Recherche d'indicateurs pertinents. Thèse INAPG, $288 \mathrm{p}$.

[43] Lavelle P. Les vers de terre de la savane de Lamto (Cote d'Ivoire) : Peuplements, Populations et Fonctions dans l'écosystème. Publication du Laboratoire de Zoologie, 1978, $12: 1-310$.

[44] Poupeau J. M. 2008. Fertilisation. L'extraordinaire pouvoir des vers de terre. Biofil. $\mathrm{N}^{\circ}$ $56,36-38$.

[45] Rafidison G.Z. Rôle de la faune dans l'humification : transformations des feuilles de hêtre par un ver anécique (Nicodrilus velox). Thèse de 3ème cycle en Agro-Eco-Pédologie, Nancy I, 1982.

[46] Doube B.M., Davoren C.W., Hindell R., Long N. \& Cass A. Surface cast production by the earthworm Aporrectodea trapezoides and the influence of organic matter on cast structural stability. In C.E. Pankhurst (Ed.), Colloquium Soil Biota: Management in sustainable farming systems, CSIRO, Australia. 1994 .

[47] Jégou D. Rôle fonctionnel de quatre espèces lombriciennes dans la structuration du sol et dans les transferts de carbone. Thèse d'Université, Rennes I., 1998, 152p.

[48] Chapuis-Lardy L., Brossard M., Lavelle P., Schouller E. Phosphorus transformations in a ferralsol through ingestion by Pontoscolex corethrurus, a geophagous earthworm. European Journal of Soil Biology., 1998, 34, 61-67. doi:10.1016/S1164-5563(99)90002-X 
[49] Orlov D.S. Publishing House of the Moscow State University, Moscow (in Russian). , 1990.

How to cite this article: Addad D., Kribaa M., Ababsa N., Tamrabet L., Hafidi M., El Fels L.end Benmahammed A. Impact of earthworm activity on the chemical fertility of irrigated soil with urban effluents. J. Fundam. Appl. Sci., 2017, 9(3), 1320-1341. 